\title{
Donor selection in the modern era
}

\author{
Kiran K. Khush \\ Division of Cardiovascular Medicine, Department of Medicine, Stanford University, Stanford, California, USA \\ Correspondence to: Kiran K. Khush, MD, MAS. Stanford Division of Cardiovascular Medicine, 300 Pasteur Drive, Falk CVRC 263, Stanford, CA, \\ 94305, USA. Email: kiran@stanford.edu.
}

\begin{abstract}
The growing disparity between the supply of donor hearts for transplantation and the demand for such organs has led to liberalization of the criteria for donor heart acceptance over the past few decades. The upper age limit and size restrictions for donor heart acceptance continue to be revised and hearts are being routinely used from donors with left ventricular dysfunction, left ventricular hypertrophy (LVH), cocaine use, multiple medical co-morbidities and after cardiopulmonary resuscitation. This article reviews recent data for use of such "expanded criteria" donor hearts and suggests ways to further increase the donor pool, including use of hearts from donors with hepatitis $\mathrm{C}$ and after circulatory determination of death. Donor biomarkers and risk scores may eventually aid in heart acceptance decisions, while ethical issues surrounding information sharing with transplant recipients remain a topic of great debate.
\end{abstract}

Keywords: Heart transplant; donor; selection; risk scores; biomarkers

Submitted Jul 31, 2017. Accepted for publication Sep 04, 2017.

doi: $10.21037 /$ acs.2017.09.09

View this article at: http://dx.doi.org/10.21037/acs.2017.09.09

\section{Introduction}

With an aging population, more patients surviving stage D heart failure and the advancement of mechanical circulatory support, there is an ever-growing and marked imbalance between the demand and supply of donor hearts for transplantation. This growing disparity between the size of heart transplant waiting lists around the globe and the availability of donor hearts for transplantation has led many transplant centers to push the boundaries of what defines a "suitable" organ. Since initial suggestions that "male cardiac donors should probably be under the age of 35 and female donors under 40 years" (1), a wealth of studies, mainly based on single-center cohorts and national registry data, have been published that have challenged prior assumptions about donor heart selection. Current guidelines for heart selection, however, remain very broad and do not address many issues encountered in routine donor selection (2), thus creating highly variable selection patterns among centers (3). Donor selection remains a challenging and multifactorial decision that is influenced by imperfect donor information (and lack of data on how to interpret this information), a potential candidate's medical urgency, donor-recipient match considerations, and institutional risk tolerance. To date, a number of consensus statements $(4,5)$ and risk models $(6,7)$ have been published in an attempt to codify knowledge around donor heart selection. What we know is that more patients are waiting for heart transplantation every year, that there is a positive association between time spent on the waitlist and mortality (8) and that the criteria used for donor heart selection must be questioned, challenged, and expanded in order to meet the evergrowing demand for heart transplantation. This Perspective discusses recent advances in the field that should help guide and expand use of available donor hearts in the modern era.

\section{Expanded use of marginal donor hearts}

Early experience with use of marginal donor hearts involved the use of alternate listing strategies, in which "marginal" donor organs were transplanted into high-risk recipients who otherwise may have been ineligible for transplantation. Use of this strategy, in which marginal donors were defined as being older and having more medical co-morbidities than standard donors, was associated with greater recipient mortality and higher resource utilization compared to 
standard heart transplant procedures but yielded a median survival over 5 years to patients who would otherwise have been expected to live for less than a year (9). Since that time, the aging donor population (which tends to have more medical co-morbidities) has resulted in abandonment of the "alternate list", with higher risk organs being accepted for the general heart transplant candidate pool. Reassuringly, recent studies have shown acceptable outcomes with these higher-risk organs, perhaps due to improved post-transplant management.

In the past $5-10$ years, analyses of large heart transplant registries has informed a cautious liberalization of donor heart acceptance criteria. For example, an analysis of the United Network for Organ Sharing (UNOS) heart transplant registry in the United States showed equivalent 30-day to 3-year survival in recipients of donor hearts without left ventricular hypertrophy $(\mathrm{LVH},<1.1 \mathrm{~cm})$, mild LVH $(1.1-1.3 \mathrm{~cm})$ and moderate-severe LVH $(\geq 1.4 \mathrm{~cm})$; however, subgroup analyses showed an increased risk of death in recipients of allografts with $\mathrm{LVH}$ and donor age $>55$ years or ischemic time $\geq 4$ hours, which highlights the need to carefully evaluate donor risk factors in aggregate (10). Donor/recipient size matching has also been relaxed. A retrospective review of 107 heart transplant procedures at the University of California-Los Angeles suggested that oversized (donor/recipient weight $\geq 1.2$ ) and undersized (donor/recipient weight $\leq 0.9$ ) donor hearts fared equally well in the setting of mild-moderate recipient perioperative pulmonary hypertension (pulmonary artery systolic pressure $\geq 40 \mathrm{mmHg}$ ) (11). However, a subsequent UNOS database study that calculated donor and recipient predicted heart mass (12) demonstrated higher mortality during the first year post-transplant (HR 1.27, $\mathrm{P}<0.001$ ) in the most undersized predicted heart mass septile, while survival did not vary across septiles of weight differences (13). This suggests that estimates of donor heart size that incorporate height, weight, age, and sex may be more useful than simply relying on weight, especially given the ever-rising prevalence of obesity across the globe. Finally, UNOS database analyses have not shown inferior post-transplant outcomes in recipients of hearts from donors who had cardiopulmonary resuscitation (even exceeding 30 minutes) (14) and from donors with past and current cocaine use (15).

Age continues to be one of the main considerations in donor heart selection. A recent analysis of data from the Spanish Heart Transplantation Registry was cautiously reassuring, demonstrating no survival disadvantage in recipients of older donor hearts (age $\geq 50$ years). However, after multivariate adjustment, recipients of older hearts did have a higher incidence of cardiac allograft vasculopathy at 5 years post-transplant (16).

There continue to be many other determinants of reduced donor heart "quality" besides the risk factors discussed above. Many transplant centers will refuse hearts with quality concerns while waiting to accept an "ideal" donor organ. This is especially true in pediatric heart transplantation, where donor acceptance criteria tend to be more stringent. Nevertheless, an analysis of recipient outcomes at a high-volume pediatric heart transplant center showed that in-hospital morbidity and long-term mortality in recipients of donor hearts refused by other centers for quality concerns were no different than outcomes in recipients of primarily offered hearts (17). More recently, an analysis of the UNOS registry showed that use of adolescent donor hearts refused by pediatric centers resulted in excellent outcomes in adult recipients (18). These studies, and others, suggest that transplant centers that have pushed the boundaries with respect to donor heart acceptance have achieved satisfactory results. The caveat, however, is that the best survival after transplantation using marginal donors hearts is seen at higher volume transplant centers (19).

\section{Donor left ventricular dysfunction}

Many donor hearts continue to be declined for transplantation due to left ventricular systolic dysfunction or regional wall motion abnormalities (20). While some donors may have a true cardiomyopathy, a significant proportion has reversible left ventricular dysfunction due to neurogenic stunned myocardium. This condition is triggered by the high catecholamine release that is typical after brain death (21).

There is convincing evidence that donor hearts with initially depressed left ventricular systolic function often improve during donor management and yield acceptable recipient outcomes after transplantation $(22,23)$. In fact, even donor hearts that continue to have reduced function at the time of organ procurement may be safely transplanted. A recent UNOS registry analysis used propensity score analysis to examine post-operative outcomes of recipients of donor hearts with left ventricular ejection fraction $<40 \%$ (reduced), 40-50\% (borderline), and $\geq 50 \%$ (normal) and showed equivalent odds of primary graft failure and death at 1 year in all 3 groups. At 1-year post-transplant, the mean ejection fraction was normal in recipients of donor hearts with reduced systolic function (24). There is certainly a concern for selection bias in such studies, as we cannot 
know the outcomes of donor hearts with left ventricular dysfunction that were declined for transplantation. However, studies such as these suggest that many donor hearts with left ventricular dysfunction can be safely used for transplantation, especially in the absence of coronary artery disease risk factors. If doubts persist, donor coronary angiography may be requested to rule out ischemic heart disease. Alternatively, Bombardini and colleagues have successfully used stress echocardiography to risk-stratify donors with left ventricular dysfunction-those that demonstrated improvement in systolic function with stress had excellent post-transplant outcomes (25), suggesting that this may be an attractive approach to identify donors with transient and reversible cardiac dysfunction.

\section{Donor risk scores}

The use of scoring systems that take multiple donor risk factors into consideration are attractive ways to evaluate donor heart offers. Such an approach has been implemented for kidney transplantation in the United States, with the goals of decreasing the discard rate of extended criteria kidneys and improving graft survival. Since 2012 UNOS has displayed the Kidney Donor Profile Index (KDPI) in its allocation software, with lower KDPI values predicting a higher long-term graft survival rate (26). That same year, Weiss and colleagues published the heart transplant donor risk index (DRI), which uses four variables to predict shortand long-term mortality after heart transplantation: donor age, blood urea nitrogen/creatinine ratio, race mismatching, and ischemic time. The utility of the DRI has been called into question as donor age is probably the only variable in this risk score that is actually related to graft quality. Ischemic time is a function of the transplant procedure, and not of the graft itself, and some centers may be willing to incur a longer ischemic time to procure a high quality donor heart. Similarly, race mismatch may affect recipient outcomes, but is unlikely to be an indicator of inherent graft quality, and the BUN/Cr ratio seems much less likely to reflect donor heart quality than measures that transplant centers routinely evaluate, such as echocardiographic findings including left ventricular function, wall thickness, and regional wall motion abnormalities. These echocardiographic variables are probably not included in the DRI because they are often missing or of questionable accuracy in the UNOS database; thus, the DRI is of limited use in the current era of routine donor echocardiography with real-time image viewing capability. Nevertheless, a recent analysis that compared recipient outcomes after transplantation with that of survival on the waiting list showed a survival benefit with transplantation compared to continued waiting, even in recipients who had higher risk donors, as defined by the DRI. This finding was most pronounced for sicker (UNOS status $1 \mathrm{~A}$ and $1 \mathrm{~B}$ ) candidates and was less apparent for stable (status 2) candidates (27).

Other groups have attempted to develop heart transplant risk scores, including a more recent UNOS database analysis by Trivedi et al. (6). Again, due to the inherent limitations of using national registry data for this purpose, the risk score encompasses many recipient risk factors but lacks input on critical donor variables of interest, including left ventricular function and wall thickness. Finally, a unique approach was recently taken by Rizzi et al., who used three intensive care scoring systems (Acute Physiology and Chronic Health Evaluation II-APACHE II, Simplified Acute Physiology Score-SAPS II, and Sequential Organ Failure Assessment-SOFA) to determine severity of illness in heart transplant donors and recipients. This study found no prognostic impact of donor-related risk scores on heart transplant outcomes (28). Indeed, the recent literature has repeatedly demonstrated that the key determinant of success in transplanting a heart has more to do with the health of the recipient than that of the donor $(20,29)$, which argues that the standard criteria used for donor heart acceptance are restricting heart transplant rates worldwide.

\section{Donor biomarkers}

The use of biomarkers represents a convenient and attractive way to risk stratify potential heart donors, as is used in acute coronary syndromes and heart failure. A heart donor biomarker should have high sensitivity and specificity to predict which hearts will have adequate hemodynamic function in the recipients, and which should not be transplanted due to higher risk of graft dysfunction.

Cardiac-specific troponins, which are well-validated in patients with myocardial infarction, have been studied for cardiac donor assessment. In potential heart donors, however, cardiac troponin I (cTnI) is universally elevated, and $29 \%$ have cTnI levels $>1 \mathrm{ng} / \mathrm{mL}$ (30). Higher troponin levels have been observed after intracranial hemorrhage rather than trauma as a cause of brain death, and high troponin levels are associated with reversible cardiac dysfunction after subarachnoid hemorrhage (31). Troponin levels appear to be influenced by the time at which they are drawn during donor management-higher levels are 
measured soon after brainstem herniation, and the levels fall subsequently, especially with improvement in cardiac function. Initial studies of elevated donor troponin levels suggested that this biomarker may predict early graft failure in the recipient $(32,33)$; however, subsequent studies failed to validate this association $(30,34)$. Currently, donor troponin levels are routinely drawn by most organ procurement organizations in the United States, and a recent UNOS database analysis of peak troponin levels measured in cardiac donors with preserved left ventricular function showed no association with primary graft failure, intermediate-term mortality, or development of cardiac allograft vasculopathy (30).

Similarly, B-type natriuretic peptide (BNP or NTproBNP) is released from the ventricular myocardium in response to increased wall stress and is elevated in the presence of cardiac dysfunction after subarachnoid hemorrhage (35). After brain death, the BNP prohormone is likely synthesized by ventricular myocytes in response to the cardiac wall stress and pressure overload that occur at the time of cerebral injury or brainstem herniation. BNP levels have been shown to be higher in potential heart donors with poor echocardiographic function (36), and NT-proBNP levels closely correlate with indices of cardiac function, by both hemodynamics and echocardiography (37).

Many challenges remain, however, in the use of biomarkers such as cTnI and BNP for assessment of potential cardiac donors. In most published studies, biomarkers were drawn at varying times after brainstem herniation, and we now know that their levels change over time during donor management. Perhaps assessment of the trend in biomarker levels during the donor management period, especially in the context of therapies administered and echocardiographic findings, may be more helpful than simply assessing a single value. Similarly, the interactions of donor and recipient characteristics with biomarker levels in donor assessment remain unknown. Prospective studies of serial donor biomarker levels at pre-determined time points during donor management, and their association with posttransplant outcomes, should shed light on their clinical utility.

\section{Hepatitis C positive donors}

Hepatitis $\mathrm{C}$ virus (HCV) infection has reached epidemic proportions and is the most common chronic blood-borne infection in the United States today. The prevalence of HCV infection in the third National Health and Nutrition
Examination Survey (NHANES III), conducted from 1988-1994 was $1.8 \%$ (38) and is likely higher today, especially in the organ donor population that has a much higher incidence of intravenous drug use than the general population. Use of hearts from HCV positive donors has been linked to development of accelerated cardiac allograft vasculopathy in the transplant recipient (39). An analysis of the US Scientific Registry of Transplant Recipients performed in 2006 showed that recipients of $\mathrm{HCV}$-positive donor hearts had increased risk of dying of liver disease and cardiac allograft vasculopathy, with an overall hazard ratio of 2.1 (40) - a finding that was confirmed with an updated analysis published in 2016 (HR 1.78 in HCV-recipients of HCV+ donor hearts) (41). Treatment of $\mathrm{HCV}$ in the transplant recipient was problematic as interferon therapy is associated with an increased risk of acute rejection and cardiotoxicity $(42,43)$. The recent development of highly effective direct-acting antiviral therapy for $\mathrm{HCV}$, however, has opened the door to use of $\mathrm{HCV}$ positive donor hearts for transplantation. Ledipasvir-sofosbuvir is an interferon-free regimen used for HCV genotypes 1a, 1b, 4, 5, and 6. Among patients with genotypes $1 \mathrm{a}$ and $1 \mathrm{~b}$ (the most common genotypes in the United States and Europe), ledipasvir-sofosbuvir is associated with a $99 \%$ sustained virologic response rate at 12 weeks (44). Preliminary results in liver and kidney transplant recipients suggest good tolerability and minimal interaction with most immunosuppressive drugs, and several heart transplant centers in the United States have recently started accepting organs from HCV+ donors. While this approach has the potential to significantly increase the supply of available donor hearts for transplantation, prospective studies will be needed to examine duration of sustained virologic response and long-term survival, as well as rates of graft loss, acute rejection, and cardiac allograft vasculopathy. The strategy of accepting hearts from $\mathrm{HCV}+$ donors merits close attention, and this data will be forthcoming. In the meantime, use of hearts from donors with Hepatitis B virus infection ( $\mathrm{HBV}$ core $\mathrm{Ab+}$ ) is increasingly common, with low risk of viral transmission in thoracic transplant recipients, and the availability of effective anti-viral prophylaxis with lamivudine or entecavir, especially for HBV non-immune recipients (45). Use of organs from donors infected with human immunodeficiency virus (HIV), on the other hand, remains restricted to research protocols in thoracic transplantation, while recent evidence from South Africa demonstrating the short-term safety of organ transplantation between HIV- 
infected persons has prompted changes to legislation in the United States to allow HIV-positive patients awaiting kidney and liver transplantation to receive organ transplants from HIV-positive deceased donors (46).

\section{Ex-vivo cardiac perfusion}

The relationship between prolonged allograft ischemic time and reduced survival after heart transplantation has been clearly demonstrated $(47,48)$, although this effect appears to be modified by donor age. This observation, which has constrained distant organ procurement, stimulated the development of technologies for continuous ex vivo perfusion of donor hearts (49). Initial work in porcine models showed that donor hearts sustained less functional impairment after storage with warm continuous perfusion, compared to cold static storage (50). The TransMedics Organ Care System (OCS) is the first and only commercially available $e x$ vivo heart perfusion platform that can maintain the donor heart in a warm, beating, near-physiological state. The OCS was recently evaluated in the Prospective MultiCenter Safety and Effectiveness Evaluation of the Organ Care System Device for Cardiac Use II (PROCEED II) clinical trial in the United States and Europe, in which 128 patients underwent heart transplantation using donor hearts randomly assigned to $e x$ vivo perfusion or standard cold storage (51). Study results showed that 30-day patient and graft survival, serious adverse events, incidence of severe rejection, and length of intensive care unit stay were similar between the two groups, thereby demonstrating non-inferiority of ex vivo perfusion during donor heart transport. Other studies have reported that ex vivo perfusion may reduce the incidence of primary graft dysfunction (52) and has been used to transport a heart with a 10-hour out of body time (53). In fact, the Harefield Hospital transplant program in London, UK routinely uses normothermic perfusion for heart transplants, with the rationale of limiting cold ischemia, particularly for redo sternotomy recipients and those undergoing complex VAD explants (54).

Although much work remains for optimizing cannulation techniques, standardizing myocardial protection (before and after use of the OCS), developing biomarkers and measures of the adequacy of myocardial perfusion and automating adjustments in perfusion, the hope is that use of ex vivo perfusion will reduce allograft preservation injury and even enable assessment and resuscitation of marginal donor hearts, thereby expanding the donor pool. The on-going TransMedics-sponsored EXPAND Heart Pivotal Trial
(https://clinicaltrials.gov/ct2/show/study/NCT02323321) aims to evaluate the safety and efficacy of the OCS for preserving and assessing 75 expanded-criteria donor hearts for transplantation at two centers in the United States. Finally, use of ex vivo perfusion systems may enable distant donor heart procurement, which could expand regional sharing beyond that currently possible.

\section{Donation after circulatory death donors}

Procurement of hearts from donors after circulatory death (DCD) represents the next frontier for increasing the availability of donor hearts for transplantation. It is estimated that DCD heart donation could increase overall heart transplant volume in the US by $15 \%$ to $23 \%$, and up to $40 \%$ for pediatric transplants (OPTN data, accessed July 21, 2017). In the United Kingdom, DCD donors represent an even greater proportion of the organ donor pool, and DCD donation could increase heart transplant rates by over $30 \%$ (55). Cardiac transplantation with distantly procured DCD hearts has only recently been undertaken, after much groundwork was performed to develop ways to minimize cardiac damage during withdrawal of life support $(56,57)$, to optimize organ preservation during transport via use of ex vivo normothermic perfusion (58) and to assess cardiac function prior to transplantation (59). The heart transplant programs at St. Vincent's Hospital (Sydney, Australia), Papworth Hospital (Cambridge, UK) and Harefield Hospital (London, UK) have pioneered the use of DCD donor hearts for transplantation, with excellent clinical outcomes. Over 45 DCD heart transplants have been performed at these three programs since February 2015, with 75-100\% 1-year survival (60).

\section{Ethical considerations}

Organ transplantation continues to be fraught with ethical considerations, including decisions regarding beneficence versus non-maleficence when considering the suitability of donor organs. The shortage of available organs required for the rising number of patients awaiting transplantation is the foundation of this debate. This organ shortage has pushed transplant physicians to broaden their criteria for organ acceptability, which may confer increased risk to the recipient. Unlike the required identification, disclosure, and recipient consent required for transplantation of Public Health Service high-risk donor organs in the United States (defined as organs from donors deemed to be at elevated risk of having transmissible viral infections, such as HIV 


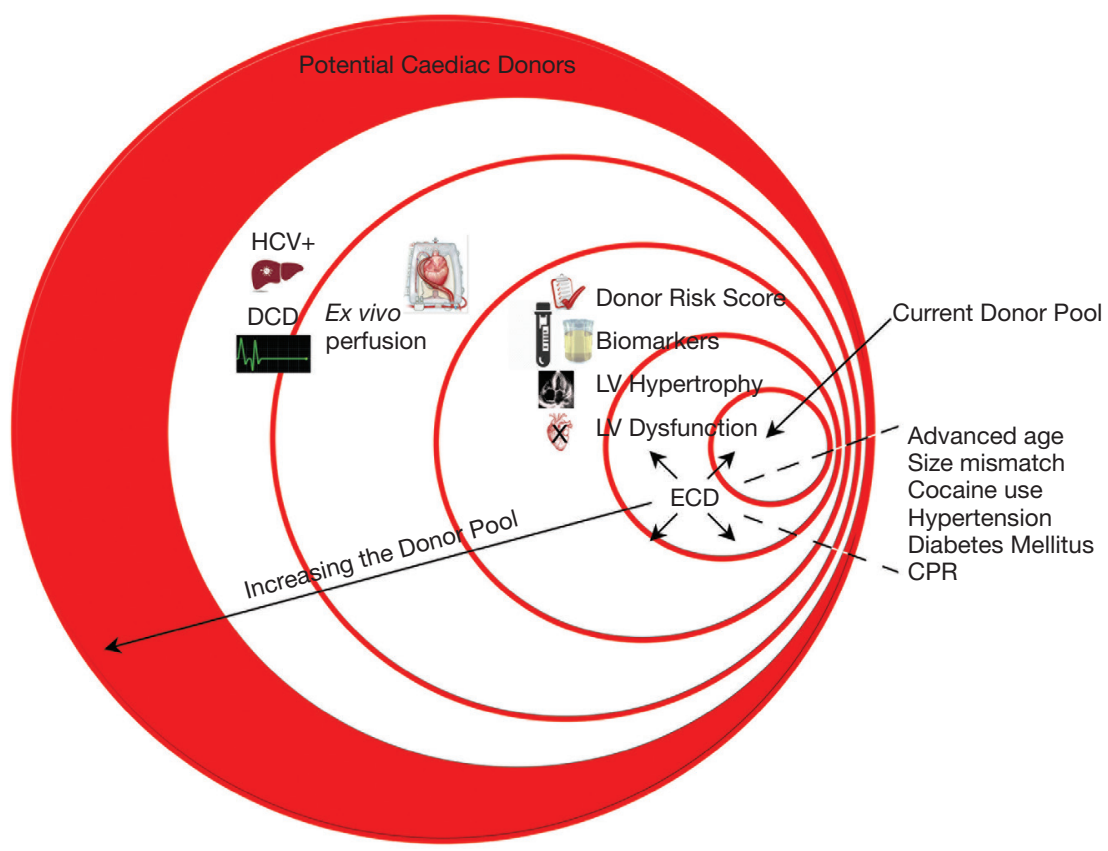

Figure 1 Potential strategies to increase the supply of donor hearts for transplantation. CPR, cardiopulmonary resuscitation; ECD, extended criteria donor; LV, left ventricular; DCD, donation after circulatory death; HCV, hepatitis C virus.

and $\mathrm{HCV}$ ), it is at the discretion of transplant centers to accept or reject organs from expanded-criteria donors and there is currently no policy requiring this information to be shared with the recipient. Similarly, an increasing number of research studies and interventions involving transplant donors are being performed, with the need to obtain informed consent from potential recipients of these organs remaining a matter of great debate (61).

\section{Conclusions}

The ever-growing demand for donor hearts for transplantation has led us to closely examine and question the criteria for "suitable" donor organs. Certainly, as the donor pool has become older, the upper age limit continues to rise, especially in Europe. Similarly, restrictions on size matching and use of hearts from donors with a history of drug abuse, hypertension, diabetes, and other medical co-morbidities continue to be relaxed. Left ventricular dysfunction is a well-recognized cardiac sequela of brain death, and donor hearts with reduced systolic function are increasingly used for transplantation, with excellent outcomes. Use of donor risk scores and biomarkers offers transplant centers ways to quantify the risk incurred with organ acceptance, while use of hearts from $\mathrm{HCV}+$ and
DCD donors represent ways to significantly increase the pool of potential organ donors (Figure 1). Going forward, prospective studies examining clinical outcomes in recipients of "higher risk" organs and development of evidence-based criteria for donor heart acceptance will hopefully improve and standardize utilization of available donor hearts for transplantation in the modern era.

\section{Acknowledgements}

Funding: The author is principal investigator of a National Institutes of Health-sponsored study on donor heart evaluation and acceptance for transplantation (R01 HL125303).

\section{Footnote}

Conflicts of Interest: The author has no conflicts of interest to declare.

\section{References}

1. Copeland JG, Stinson EB. Human heart transplantation. Curr Probl Cardiol 1979;4:1-5.

2. Costanzo MR, Dipchand A, Starling R, et al. The 
International Society of Heart and Lung Transplantation Guidelines for the care of heart transplant recipients. J Heart Lung Transplant 2010;29:914-56.

3. Khush KK, Zaroff JG, Nguyen J, et al. National decline in donor heart utilization with regional variability: 19952010. Am J Transplant 2015;15:642-9.

4. Kobashigawa J, Khush K, Colvin M, et al. Report From the American Society of Transplantation Conference on Donor Heart Selection in Adult Cardiac Transplantation in the United States. Am J Transplant 2017;17:2559-66.

5. Zaroff JG, Rosengard BR, Armstrong WF, et al. Consensus conference report: maximizing use of organs recovered from the cadaver donor: cardiac recommendations, March 28-29, 2001, Crystal City, Va. Circulation 2002;106:836-41.

6. Trivedi JR, Cheng A, Ising M, et al. Heart Transplant Survival Based on Recipient and Donor Risk Scoring: A UNOS Database Analysis. ASAIO J 2016;62:297-301.

7. Weiss ES, Allen JG, Kilic A, et al. Development of a quantitative donor risk index to predict short-term mortality in orthotopic heart transplantation. J Heart Lung Transplant 2012;31:266-73.

8. Goldstein BA, Thomas L, Zaroff JG, et al. Assessment of Heart Transplant Waitlist Time and Pre- and Posttransplant Failure: A Mixed Methods Approach. Epidemiology 2016;27:469-76.

9. Russo MJ, Davies RR, Hong KN, et al. Matching highrisk recipients with marginal donor hearts is a clinically effective strategy. Ann Thorac Surg 2009;87:1066-70; discussion 1071.

10. Wever Pinzon O, Stoddard G, Drakos SG, et al. Impact of donor left ventricular hypertrophy on survival after heart transplant. Am J Transplant 2011;11:2755-61.

11. Kwon MH, Wong S, Kittleson M, et al. Selecting oversized donor cardiac allografts for patients with pulmonary hypertension may be unnecessary. Transplant Proc 2014;46:1497-501.

12. Bluemke DA, Kronmal RA, Lima JA, et al. The relationship of left ventricular mass and geometry to incident cardiovascular events: the MESA (Multi-Ethnic Study of Atherosclerosis) study. J Am Coll Cardiol 2008;52:2148-55.

13. Reed RM, Netzer G, Hunsicker L, et al. Cardiac size and sex-matching in heart transplantation : size matters in matters of sex and the heart. JACC Heart Fail 2014;2:73-83.

14. Cheng A, Schumer EM, Trivedi JR, et al. Does Donor Cardiopulmonary Resuscitation Time Affect Heart
Transplantation Outcomes and Survival? Ann Thorac Surg 2016;102:751-8.

15. Jayarajan S, Taghavi S, Komaroff E, et al. Long-term outcomes in heart transplantation using donors with a history of past and present cocaine use. Eur J Cardiothorac Surg 2015;47:e146-50.

16. Roig E, Almenar L, Crespo-Leiro M, et al. Heart transplantation using allografts from older donors: Multicenter study results. J Heart Lung Transplant 2015;34:790-6.

17. Easterwood R, Singh RK, McFeely ED, et al. Pediatric cardiac transplantation using hearts previously refused for quality: a single center experience. Am J Transplant 2013;13:1484-90.

18. Zafar F, Rizwan R, Lorts A, et al. Implications and outcomes of cardiac grafts refused by pediatric centers but transplanted by adult centers. J Thorac Cardiovasc Surg 2017;154:528-36.e1.

19. Kilic A, Weiss ES, Allen JG, et al. Should orthotopic heart transplantation using marginal donors be limited to higher volume centers? Ann Thorac Surg 2012;94:695-702.

20. Khush KK, Menza R, Nguyen J, et al. Donor predictors of allograft use and recipient outcomes after heart transplantation. Circ Heart Fail 2013;6:300-9.

21. Mohamedali B, Bhat G, Zelinger A. Frequency and pattern of left ventricular dysfunction in potential heart donors: implications regarding use of dysfunctional hearts for successful transplantation. J Am Coll Cardiol 2012;60:235-6.

22. Venkateswaran RV, Townend JN, Wilson IC, et al. Echocardiography in the potential heart donor. Transplantation 2010;89:894-901.

23. Zaroff JG, Babcock WD, Shiboski SC. The impact of left ventricular dysfunction on cardiac donor transplant rates. J Heart Lung Transplant 2003;22:334-7.

24. Chen CW, Sprys MH, Gaffey AC, et al. Low ejection fraction in donor hearts is not directly associated with increased recipient mortality. J Heart Lung Transplant 2017;36:611-5.

25. Bombardini T, Arpesella G, Maccherini M, et al. Mediumterm outcome of recipients of marginal donor hearts selected with new stress-echocardiographic techniques over standard criteria. Cardiovasc Ultrasound 2014;12:20.

26. Rao PS, Schaubel DE, Guidinger MK, et al. A comprehensive risk quantification score for deceased donor kidneys: the kidney donor risk index. Transplantation 2009;88:231-6.

27. Nguyen VP, Mahr C, Mokadam NA, et al. The Benefit of 
Donor-Recipient Matching for Patients Undergoing Heart Transplantation. J Am Coll Cardiol 2017;69:1707-14.

28. Rizzi G, Startseva X, Wolfrum M, et al. Unfavorable Donor Pretransplant APACHE II, SAPS II, and SOFA Scores Are Not Associated With Outcome: Implications for Heart Transplant Donor Selection. Transplant Proc 2016;48:2582-7.

29. Jeevanandam V, Furukawa S, Prendergast TW, et al. Standard criteria for an acceptable donor heart are restricting heart transplantation. Ann Thorac Surg 1996;62:1268-75.

30. Madan S, Saeed O, Shin J, et al. Donor Troponin and Survival After Cardiac Transplantation: An Analysis of the United Network of Organ Sharing Registry. Circ Heart Fail 2016;9. pii: e002909.

31. Deibert E, Barzilai B, Braverman AC, et al. Clinical significance of elevated troponin I levels in patients with nontraumatic subarachnoid hemorrhage. J Neurosurg 2003;98:741-6.

32. Grant JW, Canter CE, Spray TL, et al. Elevated donor cardiac troponin I. A marker of acute graft failure in infant heart recipients. Circulation 1994;90:2618-21.

33. Potapov EV, Ivanitskaia EA, Loebe M, et al. Value of cardiac troponin I and $\mathrm{T}$ for selection of heart donors and as predictors of early graft failure. Transplantation 2001;71:1394-400.

34. Khush KK, Menza RL, Babcock WD, et al. Donor cardiac troponin I levels do not predict recipient survival after cardiac transplantation. J Heart Lung Transplant 2007;26:1048-53.

35. Tung PP, Olmsted E, Kopelnik A, et al. Plasma B-type natriuretic peptide levels are associated with early cardiac dysfunction after subarachnoid hemorrhage. Stroke 2005;36:1567-9.

36. Amir NL, Gerber IL, Edmond JJ, et al. Plasma B-type natriuretic peptide levels in cardiac donors. Clin Transplant 2009;23:174-7.

37. Dronavalli VB, Ranasinghe AM, Venkateswaran RJ, et al. N-terminal pro-brain-type natriuretic peptide: a biochemical surrogate of cardiac function in the potential heart donor. Eur J Cardiothorac Surg 2010;38:181-6.

38. Alter MJ, Kruszon-Moran D, Nainan OV, et al. The prevalence of hepatitis $\mathrm{C}$ virus infection in the United States, 1988 through 1994. N Engl J Med 1999;341:556-62.

39. Haji SA, Starling RC, Avery RK, et al. Donor hepatitis-C seropositivity is an independent risk factor for the development of accelerated coronary vasculopathy and predicts outcome after cardiac transplantation. J Heart Lung Transplant 2004;23:277-83.

40. Gasink LB, Blumberg EA, Localio AR, et al. Hepatitis C virus seropositivity in organ donors and survival in heart transplant recipients. JAMA 2006;296:1843-50.

41. Stepanova M, Locklear T, Rafiq N, et al. Long-term outcomes of heart transplant recipients with hepatitis $\mathrm{C}$ positivity: the data from the U.S. transplant registry. Clin Transplant 2016;30:1570-7.

42. Aljumah AA, Saeed MA, Al Flaiw AI, et al. Efficacy and safety of treatment of hepatitis $\mathrm{C}$ virus infection in renal transplant recipients. World J Gastroenterol 2012;18:55-63.

43. Wang BY, Chang HH, Chen IM, et al. Peginterferon alpha-2b and acute allograft failure in a heart transplant recipient. Ann Thorac Surg 2010;89:1645-7.

44. Afdhal N, Zeuzem S, Kwo P, et al. Ledipasvir and sofosbuvir for untreated HCV genotype 1 infection. N Engl J Med 2014;370:1889-98.

45. Huprikar S, Danziger-Isakov L, Ahn J, et al. Solid organ transplantation from hepatitis B virus-positive donors: consensus guidelines for recipient management. Am J Transplant 2015;15:1162-72.

46. Muller E, Barday Z, Mendelson M, et al. HIV-positiveto-HIV-positive kidney transplantation--results at 3 to 5 years. N Engl J Med 2015;372:613-20.

47. Russo MJ, Chen JM, Sorabella RA, et al. The effect of ischemic time on survival after heart transplantation varies by donor age: an analysis of the United Network for Organ Sharing database. J Thorac Cardiovasc Surg 2007;133:554-9.

48. Ford MA, Almond CS, Gauvreau K, et al. Association of graft ischemic time with survival after heart transplant among children in the United States. J Heart Lung Transplant 2011;30:1244-9.

49. Hassanein WH, Zellos L, Tyrrell TA, et al. Continuous perfusion of donor hearts in the beating state extends preservation time and improves recovery of function. J Thorac Cardiovasc Surg 1998;116:821-30.

50. Rosenbaum DH, Peltz M, DiMaio JM, et al. Perfusion preservation versus static preservation for cardiac transplantation: effects on myocardial function and metabolism. J Heart Lung Transplant 2008;27:93-9.

51. Ardehali A, Esmailian F, Deng M, et al. Exvivo perfusion of donor hearts for human heart transplantation (PROCEED II): a prospective, open-label, multicentre, randomised non-inferiority trial. Lancet 2015;385:2577-84. 
52. Koerner MM, Ghodsizad A, Schulz U, et al. Normothermic ex vivo allograft blood perfusion in clinical heart transplantation. Heart Surg Forum 2014;17:E141-5.

53. Stamp NL, Shah A, Vincent V, et al. Successful Heart Transplant after Ten Hours Out-of-body Time using the TransMedics Organ Care System. Heart Lung Circ 2015;24:611-3.

54. García Sáez D, Zych B, Sabashnikov A, et al. Evaluation of the organ care system in heart transplantation with an adverse donor/recipient profile. Ann Thorac Surg 2014;98:2099-105; discussion 2105-6.

55. Messer S, Lannon J, Wong E, et al. The Potential of Transplanting Hearts From Donation After Circulatory Determined Death (DCD) Donors Within the United Kingdom. J Heart Lung Transplant 2015;34:S275.

56. Iyer A, Chew HC, Gao L, et al. Pathophysiological Trends During Withdrawal of Life Support: Implications for Organ Donation After Circulatory Death. Transplantation
2016;100:2621-9.

57. Iyer A, Gao L, Doyle A, et al. Increasing the tolerance of DCD hearts to warm ischemia by pharmacological postconditioning. Am J Transplant 2014;14:1744-52.

58. Iyer A, Gao L, Doyle A, et al. Normothermic ex vivo perfusion provides superior organ preservation and enables viability assessment of hearts from DCD donors. Am J Transplant 2015;15:371-80.

59. Messer S, Ardehali A, Tsui S. Normothermic donor heart perfusion: current clinical experience and the future. Transpl Int 2015;28:634-42.

60. Dhital KK, Chew HC, Macdonald PS. Donation after circulatory death heart transplantation. Curr Opin Organ Transplant 2017;22:189-97.

61. Abt PL, Marsh CL, Dunn TB, et al. Challenges to research and innovation to optimize deceased donor organ quality and quantity. Am J Transplant 2013;13:1400-4.
Cite this article as: Khush KK. Donor selection in the modern era. Ann Cardiothorac Surg 2018;7(1):126-134. doi: 10.21037/ acs.2017.09.09 